\title{
(6) Advances in epilepsy surgery
}

\section{OPEN ACCESS}

${ }^{1}$ Department of Clinical and Experimental Epilepsy, UCL Institute of Neurology, London, UK

${ }^{2}$ MRI Unit, Epilepsy Society, Chalfont St Peter, UK ${ }^{3}$ Department of Neurosurgery, National Hospital for Neurology and Neurosurgery, London, UK

\section{Correspondence to}

Mark Nowell,

Department of Clinical and

Experimental Epilepsy, UCL

Institute of Neurology, Box 29,

National Hospital for

Neurology and Neurosurgery,

Queen Square, London,

WC1N 3BG, UK;

m.nowell@ucl.ac.uk

Received 21 October 2013 Revised 29 January 2014 Accepted 18 March 2014

Published Online First

9 April 2014

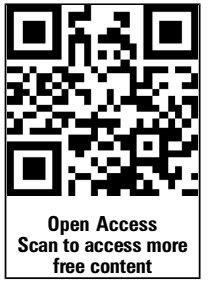

CrossMark

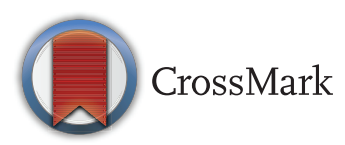

To cite: Nowell $M$, Miserocchi A, McEvoy AW, et al. I Neurol Neurosurg Psychiatry 2014;85: 1273-1279.

Mark Nowell, ${ }^{1,2,3}$ Anna Miserocchi, ${ }^{1,3}$ Andrew W McEvoy, ${ }^{1,3}$ John S Duncan ${ }^{1,2}$

\section{ABSTRACT}

This review summarises exciting recent and forthcoming advances that will impact on the surgical management of epilepsy in the near future. This does not cover the current accepted diagnostic methodologies or surgical treatments that are routinely practiced today. The content of this review was derived from a PubMed literature search, using the key words 'Epilepsy Surgery', 'Neuromodulation', 'Neuroablation', 'Advances', between 2010 and November 2013.

\section{INTRODUCTION}

Despite advances in imaging and the accumulation of neurological and surgical experience, the outcomes for seizure freedom in epilepsy surgery have not changed significantly over the last 20 years. Currently, 20-40\% of patients with epilepsy are considered refractory to medical treatment. ${ }^{1}$ Less than $50 \%$ of these are candidates for focal resective surgery, with rates of long-term seizure freedom ranging from $30 \%$ to $60 \%$ depending on the operation. ${ }^{2}$ Some argue that this apparent lack of progress is a reflection of a lowered threshold to offer surgery, and that with continued refinement of techniques, increasingly challenging cases are being taken on. However, there is widespread agreement that there remains great potential to improve nonpharmacological management, to achieve either better seizure control or complete seizure freedom.

There are three broad directions in which the next major advances may occur. First, there is the continued refinement of the current methodology. An improved hypothesis for the epileptogenic zone (EZ), based on advanced presurgical evaluation, including intracranial EEG, and thus, better patient selection for cortical resections. This is probably most relevant to sufferers of non-lesional extratemporal epilepsy, and is most likely to lead to improvements in the rates of seizure freedom. Next, there are improved surgical methods for achieving a precisely targeted cortical or subcortical resection. These can be grouped as neuroablative techniques, and include disconnection of propagation pathways, and destruction of epileptogenic foci. Neuroablation may be applied in the treatment of focal and generalised epilepsy. Third, neuromodulation may take a broader role, with the possibility of improving quality of life and being a useful palliation. This is most applicable to patients who are currently not candidates for resective epilepsy surgery, because their epilepsy arises from eloquent cortex, is multifocal or generalised.

\section{REFINEMENT OF CURRENT METHODOLOGY}

The epileptogenic index

The purpose of presurgical evaluation is to define the EZ, and to define the surrounding functional deficit zones. The notion of a single discrete area of EZ is attractive in its simplicity, although the high failure rate of resective surgery runs counter to this. Rather, it is possible that in at least some cases there are several structures involved in epileptogenesis, and a more comprehensive notion of the EZ needs to be considered.

The characteristic electrophysiological pattern of the $\mathrm{EZ}$ is the presence of high-frequency oscillations or 'rapid discharges'. Bartolomei in 2008 introduced the concept of the 'epileptogenic index (EI)', a novel quantitative measure that characterises the epileptogenicity of brain structures recorded with depth electrodes. ${ }^{3}$ The EI is based on spectral and temporal factors, with statistically high values corresponding to structures involved early in the ictal process. They found that their measure of EI effectively distinguished between mesial temporal lobe epilepsy (MTLE) and lateral neocortical epilepsy. Furthermore, in MTLE they found a statistically significant correlation between the duration of epilepsy and the number of structures disclosing high EI values, suggesting that MTLE is a gradually evolving process which progresses over time.

Visualisation of the EI, in 3D space as part of an integrated multimodal model, is the obvious next step. This would provide an alternative to the electrical source imaging, but also provide a more detailed, contoured brain map of seizure likelihood, that could be used by the surgeon to stratify the risk-benefit ratio of cortical resection. Work is already underway to make this a reality. David et al in Grenoble, report the use of statistical parametric mapping to visualise a quantification of the seizure onset zone. ${ }^{4}$ This has been applied to case studies of insular epilepsy, and also group studies on MTLE. This technique offers much promise in future research and clinical practice, with obvious applications in future guidance of stereoEEG (SEEG) implantations, and in the delineation of epileptogenic networks over time.

\section{Advances in imaging}

The rates of seizure freedom following resective surgery in sufferers of non-lesional extratemporal epilepsy remain poor. ${ }^{2}$ It is clear that better patient selection is required in these cases, with improved methods for imaging the EZ and guiding the implantation of intracranial EEG. For some time there has been interest in unmasking previously occult structural lesions, using non-routine magnetic resonance (MR) sequences and voxel-based morphometric analyses. ${ }^{5}$ There are also developmental techniques, such as EEG-fMRI, which require refinement and further evaluation in clinical practice. $^{6}$

$3 \mathrm{D}$ multimodality imaging is the simultaneous display of different structural and functional 
datasets, tailored to individual patients. The feasibility of this additional tool in a busy epilepsy surgery practice has been demonstrated, ${ }^{7}$ and a prospective study is currently underway to validate the usefulness of this in presurgical evaluation and surgical management. Ultimately, improvements in the surgical outcomes in this patient group will depend on better imaging, including visualisation of electrical abnormalities in $3 \mathrm{D}$, reliable imaging integration and robust planning and implementation of intracranial EEG (figure 1).

\section{NEUROABLATION}

There is an increasing trend in all forms of surgery towards minimally invasive techniques. This is most pertinent for neurosurgery, which often requires access to deep parts of the brain. Accurate navigation to these areas without the need for significant brain retraction has been solved by the application of stereotaxis. However, there remains the problem of how to 'execute' the surgery once instruments have been safely navigated to their targets. This problem is best framed in the context of epilepsy surgery with cortical resection following SEEG. However, it is also possible to consider patients with hippocampal sclerosis (HS), where even 'selective' amygdalohippocampectomies carry the risk of new cognitive deficit.

There are a number of interesting alternatives to 'execute' lesioning at the site of the EZ or to cause a disconnection in a minimally invasive way.

\section{Radiofrequency (RF) thermocoagulation}

There is much interest in complementing the technique of SEEG with a therapeutic component to lesion cortex that is sampled by the electrodes. The most obvious solution is by thermocoagulation, using a RF generator connected to the electrode contacts. A feasibility study from Lyons described this technique in 2004 , in 20 patients undergoing SEEG implantation. ${ }^{8}$

There are several benefits with this technique. It builds on the SEEG method that is well established, and proven to be safe and reliable. It is well tolerated by the patient and does not require general anaesthesia. Multiple sites can be lesioned, with realtime clinical and electrophysiological feedback. Finally, this method does not preclude the possibility of subsequent conventional open surgery.

One disadvantage with this technique is that there is no realtime feedback on the lesioning process with regards to local temperatures. The operator relies on an abrupt decrease in current to indicate coagulation of surrounding cortex. Also, RF thermocoagulation is known to be an inherently imprecise mode of thermal energy delivery, with theoretical risk to surrounding structures.

Overall, the results for SEEG and thermocoagulation are modest. A case series of 41 patients from the Lyons group report that $20(48.7 \%)$ experienced a significant decrease in seizures of at least 50\%, and $21(51.3 \%)$ did not benefit from the procedure. ${ }^{9}$ Only one patient was seizure-free following the procedure. There were no reports of worsening seizures. These results suggest that RF thermocoagulation may be a low-risk, palliative procedure, which can be considered as first-line treatment in patients undergoing SEEG to improve seizure control. It seems that this technique is particularly suited to patients for whom conventional surgery is contra-indicated or considered too high risk, such as patients with deep epileptogenic heterotopic nodules. However, a randomised controlled trial is needed to determine which patient group exactly is most likely to benefit from RF thermocoagulation.

\section{MR-guided focused ultrasound}

Magnetic resonance-guided focused ultrasound surgery (MRgFUS) is an accurate method of delivering high doses of transcranial ultrasound energy to a discrete intracranial focal point. ${ }^{10}$ The major historical barrier to this method was the need to create a craniectomy window prior to treatment, to prevent the 'defocusing' effect of the skull. However, recent advances in phased array transducer technology have overcome this defocusing effect, so that the treatment can be administered in a 'closed' method without the need for conventional surgery.

The MRgFUS consists of a clinical 3 T MRI, with a transcranial hemispheric array transducer that has 1024 ultrasound elements. The patient's head is fixed to the system in a stereotactic frame and the transducer is filled with degassed water to allow ultrasound waves to propagate toward the patient's head. Treatment planning is based on MRI, and MR thermometry is used for target verification during the procedure. The treatment can be administered on an outpatient basis, and treatment effect can be monitored by postoperative MRI (figure 2).

MRgFUS has previously been used to execute a selective medial thalamotomy in the treatment of chronic neuropathic pain. ${ }^{11}$ A US food and drug administration (FDA)-approved phase I trial using MRgFUS thalamotomy in the treatment of essential tremor has just been completed, and showed clinical improvements in 15 patients. ${ }^{12}$ There are plans for further trials in the treatment of metastatic brain tumour and Parkinson's disease.

MRgFUS has obvious and compelling attractions in epilepsy surgery. There is the avoidance of any latency period or the risk of secondary tumours with ionising radiation, which comes with radiosurgery. There is the convenience of the treatment, which does not involve any skin incision and, therefore, avoids the surgical risks of infection, haemorrhage and wound dehiscence. There are no trajectory restrictions and, crucially, there is near-real-time feedback of the lesioning effect, with MRI thermometry.

The main concern with MRgFUS is the risk of inadvertent heating of the skull base and critical structures such as cranial nerves, which results from the 'shadow' effect of energy distal to the focal point of the target. Cadaveric studies have yielded techniques to minimise this collateral heating, by building into the system software certain 'no pass' areas at the base of the brain. ${ }^{13}$ However, this remains a significant barrier (at present) to the use of MRgFUS to lesion cortical and subcortical targets. In theory, MRgFUS should evolve to become an important treatment modality in epilepsy surgery, although it is important to note there are no current cadaveric or clinical trials underway to determine efficacy.

\section{Laser ablation}

Ablation can also be achieved by MRI-guided laser interstitial thermal therapy (MRgLITT). The commercially available Visualase Thermal Therapy System combines a $15 \mathrm{~W} 980 \mathrm{~nm}$ diode laser and cooled laser application system with an imageprocessing workstation. The applicator is inserted to reach the target by a stereotactic method, and laser treatment is applied in the MR scanner, with MR thermal imaging to visualise the thermal ablation.

MRgLITT avoids the complications associated with radiosurgery. The ablation is more precise than that achieved with RF thermocoagulation, and has reliable real-time feedback. Furthermore, it appears to avoid the heating of the skull base seen in ultrasound ablation. MRgLITT is a stereotactic surgical 

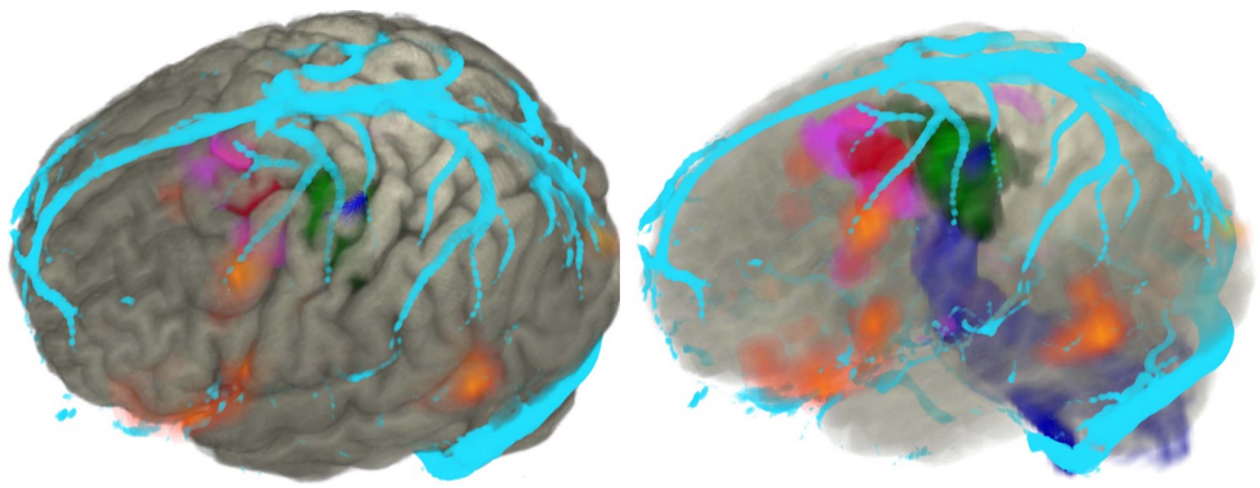

Figure 1 Volume rendering of cortex (grey) displayed in AMIRA with the following associated modalities: focal cortical dysplasia (red), fluorodeoxy glucose positron emission tomography (FDG-PET). hypometabolism (purple), hand motor fMRI (green), corticospinal tractography (blue), veins (cyan).

procedure, however, and therefore carries the surgical risks of haemorrhage and infection.

This technology has recently received FDA clearance for ablation in neurosurgery, and has previously been reported in the treatment of brain metastases. ${ }^{14}$ A recent study describes the initial use of this technique in the treatment of focal epilepsy in five children. ${ }^{15}$ Lesions included a cingulate tuber, HS, hypothalamic hamartoma $(\mathrm{HH})$ and focal cortical dysplasia. There were no complications, and early experience indicates that this is a safe procedure. All patients were seizure-free at the time of going to print, but follow-up is short and no meaningful information can be drawn on long-term efficacy at present. A pilot study is currently underway, which will examine longer-term seizure outcomes in 20 patients, It is our view that MRgLITT is an exciting prospect, which is closer to clinical adoption in epilepsy surgery than is MRgUS.

\section{Stereotactic radiosurgery}

Stereotactic radiosurgery (SRS) is a well-established technique that uses focused ionising radiation to target deep-seated lesions, sparing damage to surrounding tissue. The ionising radiation breaks chemical bonds and results in the production of free radicals. Ionising radiation can be generated by proton beam accelerators and photon accelerators. The most widely used sources of ionising radiation are photon accelerators, such as Cyberknife and Gamma Knife.

The main advantage of SRS is that deep-seated and multiple lesions can be treated without any surgical approach, avoiding

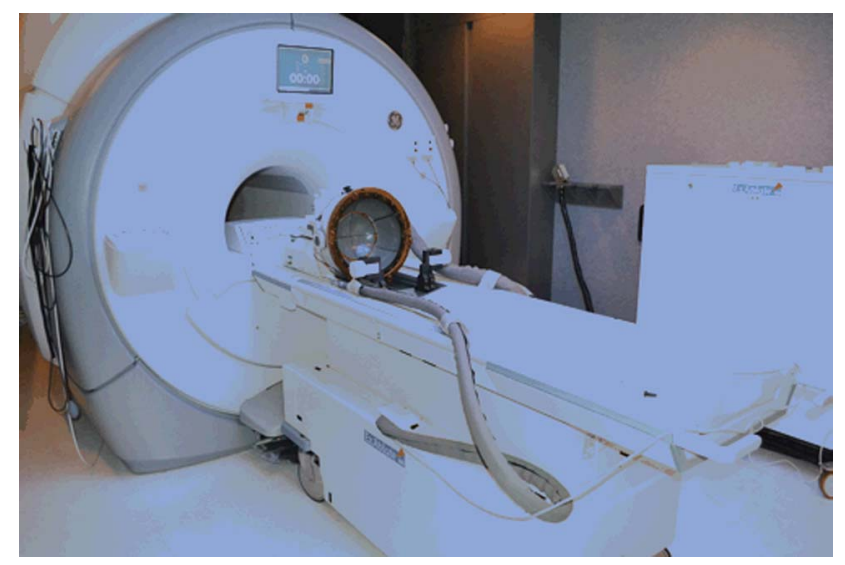

Figure 2 Commercially available magnetic resonance-guided (MR-guided) focused ultrasound. ${ }^{10}$ the inherent brain retraction/injury. The main disadvantages include the latent period of efficacy, collateral tissue injury secondary to radiation, and late-onset secondary malignancies. Furthermore, the efficacy of SRS in the treatment of different conditions is not fully understood.

The anticonvulsant effects of SRS were first observed in the treatment of tumours and vascular lesions. ${ }^{16}$ SRS has also been used as a disconnection technique in the treatment of generalised epilepsy by corpus callosotomy. ${ }^{17}$ Current interest is centred mainly on the treatment of HHs and HS.

\section{Hypothalamic hamartoma}

SRS is increasingly being considered for the treatment of $\mathrm{HH}$. Treatment is best done in early childhood, before the development of secondary generalised seizures, behavioural problems and developmental delay. In selected cases, conventional open surgery offers higher rates of seizure freedom, with the pooled results from multiple studies showing seizure freedom rates from open surgery at $50 \%$ and SRS at $30-40 \% .{ }^{18}{ }^{20}$ However, depending on the characteristics and location of the $\mathrm{HH}$, open surgery may be considered too hazardous, and SRS is the obvious alternative. ${ }^{21}$ SRS can also be used in conjunction with open surgery in patients with large $\mathrm{HH}$, if surgical debulking leaves an unresectable residual epileptogenic intrahypothalamic component. $^{22}$

\section{Hippocampal sclerosis}

The use of SRS in the treatment of HS is controversial, since conventional anterior temporal lobe resections offer a proven and reliable treatment method. The theoretical advantage of SRS is that the EZ may be lesioned in a selective way, without injury to the lateral neocortex and corresponding neuropsychological complications.

The results of an early prospective multicentre trial on the efficacy of SRS in the treatment of MTLE were promising, with seizure outcome at 2 years comparable to that of standard surgery. ${ }^{23}$ No significant cognitive deficits were seen and, in fact, $20 \%$ experienced some degree of cognitive improvement. This compares favourably to standard surgery in which cognitive impairment, particularly memory and word-finding, is observed in 30\%, and improvements are seen in $10-20 \% .{ }^{24}$

A further multicentre prospective trial in the USA randomised patients to SRS with high (24 Gy) or low (20 Gy) dose delivered to the targets. ${ }^{25}$ At 3 years, seizure freedom was $77 \%$ in the high dose and 59\% in the low dose group. Again, the neuropsychological profiles compared favourably with results from conventional surgery. 
Despite these results, there remain questions on the use of SRS in MTLE. There are a number of published series that do not give the same efficacy rates in terms of seizure outcome. ${ }^{26-}$ ${ }^{28}$ Interpretation of these is complicated by differences in protocol, including dose, isodose centres or 'shots', and volume, but the discordance in results should not be ignored.

The rate of optic radiation injury appears to be similar to that seen in conventional open surgery, with homonymous field defects seen in $43-50 \% . .^{23} 25$ This is a significant risk in those patients who become seizure-free and aspire to gain a driving license, and may compare poorly with the results of open surgery in which intraoperative visualisation of optic radiation tractography may be employed ${ }^{29}$ and reduce the risk of visual field defects.

Finally, there are unique risks with SRS that are not seen with conventional surgery, which may be related to the latent time course. Progressive radiological changes are observed, with the development of dose-dependent T2 hyperintensity, contrast enhancement and vasogenic oedema, with mass effect at 9 months postoperatively, and peaking at 12 months. These changes correspond to declines in complex partial seizures and transient increases in auras; $70 \%$ of patients in the Barbaro study also report new onset headaches postoperatively, although the timing of these is not predictable. ${ }^{25}$

A National Institutes of Health (HIF)-funded multicentre randomised controlled trial, the Radiosurgery or Open Surgery for Epilepsy (ROSE) trial, was designed to answer these outstanding questions. The trial randomised patients with MTLE to conventional surgery or SRS, and was to compare seizure outcome, cognitive outcome, quality of life and cost with an initial 3-year follow-up. Unfortunately, recruitment to this trial has currently stopped, with poor recruitment cited as the reason, and the continued funding of the work is in doubt. Without class 1 evidence, the relative merits of these two treatment paradigms will likely remain unclear.

\section{Extratemporal epilepsies}

There are no reports on the use of SRS in non-lesional extratemporal epilepsy. Certainly, the prerequisite need for intracranial implantation to determine the $\mathrm{EZ}$ would negate the main benefit of SRS as a non-invasive procedure. However, since other ablative methods have their own disadvantages, and so far produce only modest results, SRS should not be completely dismissed as a possibility.

\section{NEUROMODULATION}

Functional neurosurgery refers to the surgical manipulation of brain behaviour by the stimulation or removal of a population of neurones. The most successful application of functional neurosurgery is in the stimulation treatment of movement disorders such as idiopathic Parkinson's disease and essential tremor. However, there are also applications for chronic pain disorders, psychiatric disorders and epilepsy.

In this context, resective epilepsy surgery may be considered under the umbrella term, functional neurosurgery, since it involves the removal of a population of cells in the EZ. However, conventionally functional neurosurgery in epilepsy refers to the stimulation of cell populations, either through a cranial nerve or directly through an implanted electrode.

\section{Cranial nerve stimulation}

Vagal nerve stimulation

Vagal nerve stimulation (VNS) is a well-established palliative treatment for epilepsy, in patients who are not candidates for resective surgery. Although VNS is unlikely to offer any advance in epilepsy surgery, the elucidation of the mechanism of action may have important consequences for other related treatments. Current evidence points towards a deactivation of the nucleus of the solitary tract, with widespread projections to the dorsal raphe nucleus, locus coeruleus, hypothalamus, thalamus, amygdala and hippocampus. ${ }^{30}$

\section{Trigeminal nerve stimulation}

Trigeminal nerve stimulation (TNS) is similar to VNS as it involves the widespread stimulation of brain nuclei from an afferent cranial nerve. Like VNS, the mechanism of action is not well understood, but is thought to involve deactivation of the nucleus of the solitary tract and the locus coeruleus.

TNS differs from VNS in several important ways. First, TNS does not require surgical implantation, but can be delivered in a flexible and disposable way by application of electrodes to the forehead to stimulate the supra-orbital nerves. This confers a significant advantage, offering patients a trial of treatment to assess whether they are likely to be responders, without exposure to the surgical risks of implantation. This also opens up the opportunity for structural and functional MR studies in patients undergoing TNS.

Second, the type of stimulation offered by TNS differs from VNS, as it is high frequency and bilateral. In animal models, this type of stimulation has been shown to be superior, with increased magnitude of the seizure-reduction effect. ${ }^{31}$

A randomised controlled trial of TNS in drug-resistant epilepsy has provided preliminary evidence that this is a safe and effective treatment, with a significant within-group improvement in responder rate over time, defined as a $50 \%$ reduction in seizure frequency seen at 18 weeks. ${ }^{32}$ However, no significant differences were seen between groups, due to the high responder rate in the control group. No patients were rendered seizure-free with this treatment. Large-scale phase III multicentre trials are required to better understand the efficacy of TNS, and also to optimise treatment parameters. There are a number of variables that can be manipulated, including frequency, amplitude and duration of stimulation. Despite this, the future role for TNS is likely to be as a more tailored, safer alternative to VNS; it may offer the twin benefits of improved seizure control and improvements in mood, but is unlikely to be a curative treatment.

\section{Deep brain stimulation}

There is a long history of interest in the use of deep brain stimulation (DBS) for epilepsy control. The postulated mechanism of action is by interrupting the propagation of seizure activity, or by increasing the overall seizure threshold. Multiple targets have been put forward, centred in and around the circuit of Papez. Wu et al have provided an excellent summary of DBS targets for the treatment of epilepsy ${ }^{33}$ (table 1).

The current results with DBS for the treatment of epilepsy remain modest, even accounting for the difficult patient group with highly refractory epilepsy. Stimulation-related side effects have been reported, most commonly with psychiatric disturbances and depression. There is also the possibility of habituation to long-term stimulation. Taken together, these considerations have stimulated interest in a closed-loop, responsive form of neurostimulation, which only administers stimulation if triggered by seizure activity.

\section{Closed-loop stimulation}

The mechanism of action for closed-loop stimulation (CLS) is similar to DBS, with a rising of seizure threshold or inhibition 
Table 1 Table to show the summary of targets for DBS for the treatment of epilepsy

\begin{tabular}{|c|c|c|c|}
\hline \multirow[b]{2}{*}{ Target } & \multicolumn{2}{|l|}{ Controlled trial } & \multirow[b]{2}{*}{ Outcome } \\
\hline & Primary author & Year & \\
\hline Anterior nucleus of the thalamus & Fisher & 2010 & SANTE trial: $40.4 \%$ median seizure reduction \\
\hline Cerebellum & $\begin{array}{l}\text { Van Buren } \\
\text { Wright } \\
\text { Velasco }\end{array}$ & $\begin{array}{l}1978 \\
1984 \\
2005\end{array}$ & $\begin{array}{l}69 \% \text { seizure reduction in } 80 \% \text { patients } \\
\text { Ineffective } \\
>50 \% \text { seizure reduction in } 80 \% \text { cases }\end{array}$ \\
\hline Centromedian nucleus of the thalamus & $\begin{array}{l}\text { Fisher } \\
\text { Velasco }\end{array}$ & $\begin{array}{l}1992 \\
2000\end{array}$ & $\begin{array}{l}>50 \% \text { seizure reduction in } 80 \% \text { cases } \\
\text { No significant difference }\end{array}$ \\
\hline Hippocampus & $\begin{array}{l}\text { Tellez-Zenteno } \\
\text { Velasco } \\
\text { McLachlan }\end{array}$ & $\begin{array}{l}2006 \\
2007 \\
2010\end{array}$ & $\begin{array}{l}15 \% \text { seizure reduction } \\
>50 \% \text { seizure reduction in all } 9 \text { patients } \\
33 \% \text { seizure reduction }\end{array}$ \\
\hline Caudate nucleus & None (case reports only) & & \\
\hline Subthalamic nucleus & None (case reports only) & & \\
\hline Corpus callosum/fornix & None (animal models only) & & \\
\hline Posterior hypothalamic mammillary nuclei & None (case reports only) & & \\
\hline Locus coeruleus & None (case reports only) & & \\
\hline
\end{tabular}

of propagation. Targets for stimulation depend on the accurate localisation of the EZ, which is a prerequisite to implantation. This technique is most appropriate for patients with bitemporal or multifocal epilepsy, with no more than two distinct EZ. It also complements the technique of SEEG in cases where the EZ is localised to eloquent cortex with considerable risks with resection.

CLS relies on a robust form of automated seizure detection. The most popular methods are based on analysis of EEG waveforms across the time domain, and include the following:

- line length tool, to detect changes in frequency

- area tool, to detect changes in signal amplitude

- half-wave tool, to detect the number of waves that exceed a predetermined amplitude and duration.

There does not appear to be any single superior method for seizure detection. Instead, a tailored approach seems most promising, using combinations of these tools modified and optimised for individual patients. ${ }^{34}$

The RNS System Pivotal Clinical Investigation was a multicentre, randomised, double-blind sham stimulation controlled trial in 191 patients with refractory epilepsy ${ }^{35}$ During the initial 12-week blinded evaluation period, an initial implantation effect was noted, with a reduction in seizure frequency in the stimulation and sham groups of $34.2 \%$ and $25.2 \%$, respectively. This implantation effect was thought to be a placebo effect, or an effect of the anaesthesia. However, as the blinded period progressed, the improvement in seizure frequency persisted and improved in the stimulation group to $41.5 \%$, while improvement in the sham group fell by $9.4 \%$. At 2 years median, seizure frequency was reduced by more than $50 \%$ with a responder rate of greater than $45 \%$ with stimulation. Additionally, there were significant improvements in quality of life reported by patients receiving stimulation, via the Quality of life in Epilepsy Inventory (QOLIE-89). Of particular interest, there was no reported worsening of neuropsychological conditions or mood disorders that were commonly seen in conventional DBS.

Clearly, CLS requires more study, with outstanding questions on seizure detection algorithms, targets and stimulation parameters, and long-term follow-up. However, this remains an exciting prospect as a further treatment modality in refractory epilepsy (figure 3).
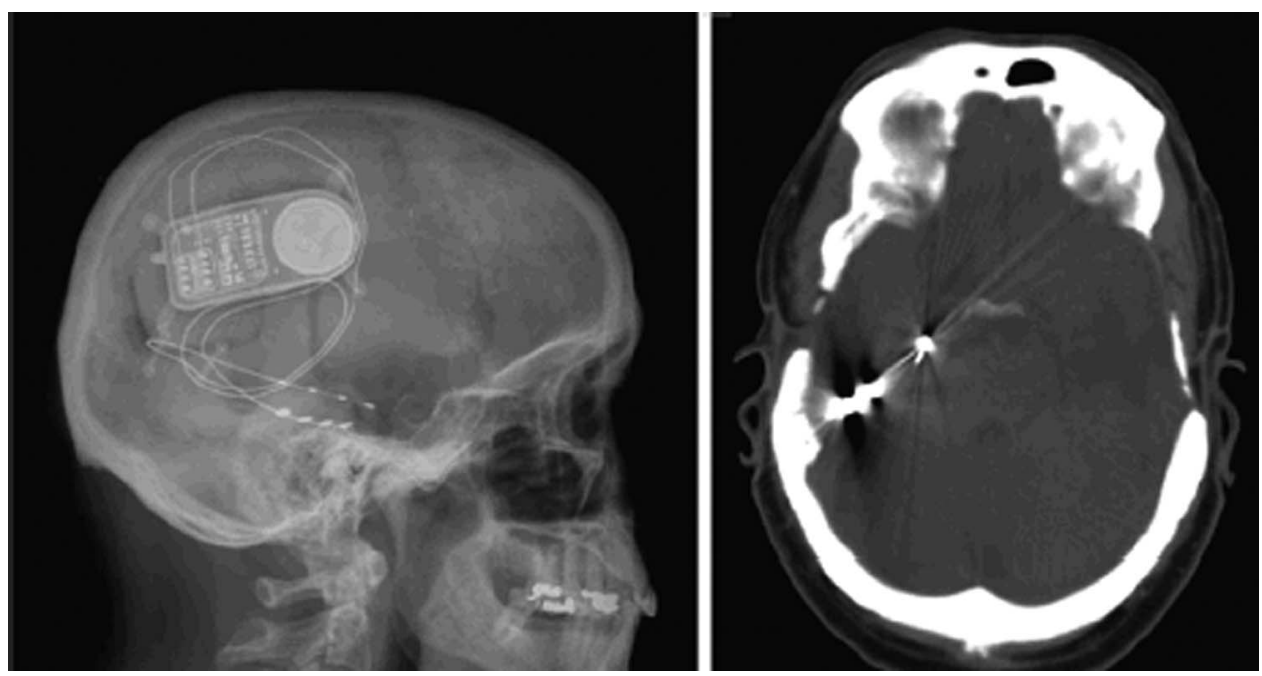

Figure 3 Closed-loop stimulation, with a depth electrode in the right hippocampus and a subdural grid over the inferior surface of the temporal lobe. ${ }^{35}$ 


\section{Closed-loop local drug delivery}

It is an attractive possibility that localised intracerebral delivery of antiepileptic drugs (AED) can improve the efficacy of pharmacological treatment of epilepsy, without systemic side effects ${ }^{36}$ Several groups are, therefore, engaged in research developing automated local drug delivery systems, comprising of seizure detection technology coupled with intracranial delivery of $\mathrm{AED}^{37}$ The most promising of these is the subdural hybrid neuroprosthesis (HNP), described by Ludvig. Safety and efficacy is currently being investigated in rat and monkey models, but there remains some distance to go before human clinical trials. Conceptually, this is closely related to the closed-loop systems of neurostimulation. However, this is arguably a more challenging path to take, requiring close collaboration between the disciplines of microengineering, neuropharmacology, neurophysiology and neurosurgery.

\section{SUMMARY}

There are several exciting avenues for further technological advances in the surgical treatment of epilepsy surgery.

For patients undergoing open surgery, improving their outcomes will depend on the stepwise refinement of current methodology, with advances in imaging epilepsy paramount. For patients who are currently not candidates for open surgery, increased delivery of treatment options by minimally invasive techniques, either neuromodulatory or ablative in nature, is likely to occur in the future.

Advances in neuromodulation will coincide with the elucidation of the mechanism of action, and much work needs to be done to refine individual stimulation targets and stimulation parameters. New neuromodulation techniques will also require a robust evidence base for clinical indications, long-term efficacy and safety. As described, there are real difficulties in designing trials for new epilepsy treatments where the findings of high responder rates in control groups are a common feature and compromise the interpretation of studies. One reason for control responses is the well-documented placebo effect, which is often present following functional sham surgery. Other possible causes, seen in drug trials and trials of new surgical treatments, are related to the selection of the control group. The group can be compromised by the phenomenon of 'regression towards the mean', where inconsistency in seizure frequency, and high initial seizure frequency, can later give the false impression of response rates in the control group as the study progresses. Additionally, it is not uncommon for patients in control groups to have their medical treatment optimised, with a consequent improvement in seizure control. Finally, outcome is a complex term in epilepsy surgery, encompassing the interplay of seizure freedom, seizure control, cognitive and surgical morbidity and quality of life over a prolonged period of time. In the future, large-scale and well-designed randomised controlled controls will have to take these factors into account to provide the evidence base for clinical implementation.

There is no consensus on the most promising technique for neuroablation, and competition between different methods will continue. In terms of clinical implementation, there are already centres that use SRS and SEEG-guided RF routinely, while MRgLITT is undergoing early clinical trials and MRgFUS remains very much a research tool in epilepsy. One major disadvantage of neuroablation in general is that the size of the lesioning is limited, and large, effective 'resections' can only be achieved by the repeated lesioning of different contiguous targets. For SEEG-guided RF and MRgLITT, this entails repeated passage of hardware through the brain, with associated risk of vascular injury. The precise targeting of individual propagation pathways and epileptogenic foci is, therefore, likely to be a largely palliative measure, with the eventual emergence of previously masked pathways and foci to continue seizure propagation following surgery. Unfortunately, this may be an insurmountable limitation with neuroablation when compared with conventional open surgery and cortical resection.

Finally, epilepsy surgery remains a significantly underused resource. It is often perceived as a treatment of last resort, with patients typically referred after 20 years of seizures. ${ }^{38}$ This contrasts with the NICE (National Institute of Health and Care Excellence) guidelines, which recommend referral to a tertiary service if epilepsy is not controlled within 2 years (NICE clinical guideline 137, 2012). ${ }^{39}$ This also runs counter to the evidence that epilepsy surgery is a cost-effective treatment, with large savings in seizure-free patients, as anticonvulsants and hospital admissions are successfully eliminated. ${ }^{40}$ Perhaps the most important advance for the future would be to increase awareness in the general population, and education among health professionals, on the safety and efficacy of epilepsy surgery as an early intervention in medically refractory focal epilepsy.

Early referrals to tertiary centres, coupled with the rigorous application of systematic presurgical evaluation pathways in a multidisciplinary environment, and with 3D multimodality imaging, may be the simplest and surest way to advance epilepsy surgery in the near future.

Acknowledgements JSD has received institutional grant support from Eisai, UCB Pharma, GSK, Janssen Cilag, Medtronic, and GE Healthcare. AM has received support from UCB, Baxter, and Cyberonics. The remaining authors have no conflicts of interest. We confirm that we have read JNNP's position on issues involved in ethical publication and affirm that this report is consistent with those guidelines.

Contributors MN conceived the review, performed the literature search and produced the first draft, as a chapter for a thesis entitled 'Novel Multimodality Imaging in Epilepsy Surgery'. AM helped with design, revised the draft with special consideration to the Neuromodulation section, and gave final approval. A McEvoy helped with design, revised the draft with special attention to the Neuroablation section, and gave final approval. JSD conceived the idea of submitting the chapter as a review, revised the entire draft with special consideration to the Advances in Imaging section, and gave final approval.

Funding MN is supported by Wellcome Trust/Dept of Health Programme Grant (HICF 4-275). We are grateful to the Wolfson Trust and the Epilepsy Society for supporting the Epilepsy Society MRI scanner. This work was undertaken at UCLH/ UCL, who received a proportion of funding from the Department of Health's NIHR UCLH Biomedical Research Centre funding scheme.

\section{Competing interests None.}

\section{Patient consent Obtained.}

Ethics approval This review forms part of a project, approved by the Joint Research Ethics Committee of the National Hospital for Neurology and Neurosurgery (NHNN), and University College London (UCL) Institute of Neurology (ION) and by other relevant institutional ethics committees.

Provenance and peer review Not commissioned; externally peer reviewed.

Open Access This is an Open Access article distributed in accordance with the terms of the Creative Commons Attribution (CC BY 3.0) license, which permits others to distribute, remix, adapt and build upon this work, for commercial use, provided the original work is properly cited. See: http://creativecommons.org/licenses/by/3.0/

\section{REFERENCES}

1 Tellez-Zenteno JF, Dhar R, Wiebe S. Long-term seizure outcomes following epilepsy surgery: a systematic review and meta-analysis. Brain 2005;128:1188-98.

2 De Tisi J, Bell G, Peacock J, et al. The long-term outcome of adult epilepsy surgery, patterns of seizure remission, and relapse: a cohort study. Lancet 2011;378:1388-95.

3 Bartolomei F, Chauvel P, Wendling F. Epileptogenicity of brain structures in human temporal lobe epilepsy: a quantified study from intracerebral EEG. Brain 2008;131:1818-30.

4 David 0, Blauwblomme T, Job A, et al. Imaging the seizure onset zone with stereo-electroencephalography. Brain 2011;134:2898-11. 
5 Focke NK, Bonelli SB, Yogarajah M, et al. Automated normalized FLAIR imaging in MRI-negative patients with refractory focal epilepsy. Epilepsia 2009;50:1484-90.

6 Ziljmans M, Huiskamp G, Hersevoort M, et al. EEG-fMRI in the pre-operative work-up for epilepsy. Brain 2007;130:2343-53.

7 Rodionov R, Vollmar C, Nowell M, et al. Feasibility of multimodal 3D neuroimaging to guide implantation of intracranial EEG electrodes. Epilepsy Res 2013; 107:91-100.

8 Guenot M, Isnard J, Ryvlin P, et al. SEEG-guided RF Thermocoagulation of Epileptic Foci: feasibility, safety and preliminary results. Epilepsia 2004;45:1368-74.

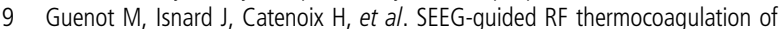
epileptic foci: a therapeutic alternative for drug-resistant non-operable partial epilepsies. Adv Tech Stand Neurosurg 2011:36:61-78.

10 Monteith S, Sheehan J, Medel R, et al. Potential intracranial applications of magnetic resonance-guided focused ultrasound surgery. J Neurosurg 2012;23:1-7.

11 Martin E, Jeanmonod D, Morel A, et al. High-intensity focused ultrasound for noninvasive functional neurosurgery. Ann Neurol 2009;66:858-61.

12 Elias WJ, Huss D, Voss T, et al. A pilot study of focused ultrasound thalamotomy for essential tremor. N Eng J Med 2013;369:640-8.

13 Monteith S, Sheehan J, Medel R, et al. Potential intracranial applications of magnetic resonance-guided focused ultrasound surgery. J Neurosurg 2013:118:215-21.

14 Carpentier A, McNichols R, Stafford R, et al. Real-time magnetic resonance guided laser thermal therapy for focal metastatic brain tumours. Neurosurgery 2008;63 (Suppl 1):21-8.

15 Curry D, Gowda A, McNichols R, et al. MR-guided stereotactic laser ablation of epileptogenic foci in children. Epilepsy Behav 2012;24:408-14.

16 Quigg M, Rolston J, Barbaro N. Radiosurgery for epilepsy: clinical experience and potential antiepileptic mechanisms. Epilepsia 2012;53:7-15.

17 Bodaghabadi M, Bitaraf MA, Aran S, et al. Corpus callosotomy with gamma knife radiosurgery for a case of intractable generalised epilepsy. Epileptic Disord 2011;13:202-8.

18 Frazier JL, Goodwin CR, Ahn ES, et al. A review on the management of epilepsy associated with hypothalamic hamartomas. Child Nerv Syst 2009;25:423-32.

19 Regis J, Bartolomei F, de Toffol B, et al. Gamma knife surgery for epilepsy related to hypothalamic hamartomas. Neurosurgery 2000;47:1343-51.

20 Pati S, Sollman M, Fife TD, et al. Diagnosis and management of epilepsy associated with hypothalamic hamartoma: an evidence based systemic review. J Child Neurol 2013;28:909-16.

21 Rosenfeld J, Feiz-Erfan I. Hypothalamic hamartoma treatment: surgical resection with the transcallosal approach. Semin Pediatr Neurol 2007;14:88-98.

22 Romanelli $\mathrm{P}$, Striano $\mathrm{P}$, Barbarisi $\mathrm{M}$, et al. Non-resective surgery and radiosurgery for treatment of drug-resistant epilepsy. Epilepsy Res 2012;99:193-201.
23 Regis J, Rey M, Bartolomei F, et al. Gamma knife surgery in mesial temporal lobe epilepsy: a prospective multicentre study. Epilepsia 2004;45:504-15.

24 Baxendale S, Thompson P, Duncan J. Improvements in memory function following anterior temporal lobe resection for epilepsy. Neurology 2008:71:1319-25.

25 Barbaro NM, Quigg M, Broshek D, et al. A multicentre prospective pilot study of gamma knife radiosurgery for mesial temporal lobe epilepsy: seizure response, adverse events and verbal memory. Ann Neurol 2009;65:167-75.

26 Romanelli P, Muacevic S, Striano S. Radiosurgery for hypothalamic hamartomas. Neurosurg Focus 2008;24:E9.

27 Hoggard N, Wilkinson I, Griffiths P, et al. The clinical course after stereotactic radiosurgical amygdalohippocampectomy with neuroradiological correlates. Neurosurgery 2008;62:336-44.

28 Vojtech J, Vladyka V, Kalina M, et al. The use of radiosurgery for the treatment of mesial temporal lobe epilepsy and long-term results. Epilepsia 2009:50:2061-71.

29 Winston GP, Daga P, Stretton J, et al. Optic radiation tractography and vision in anterior temporal lobe resection. Ann Neurol 2012;71:334-41.

30 Fornai F, Ruffoli R, Giorgi FS, et al. The role of locus coeruleus in the antiepileptic activity induced by vagal nerve stimulation. Eur J Neurosci 2011;33:2169-78.

31 DeGeorgia C, Fanselow E, Schrader L, et al. Trigeminal nerve stimulation: seminal animal and human studies for epilepsy and depression. Neurosurg Clin N Am 2011:22:449-56.

32 DeGiorgia C, Soss J, Cook I, et al. Randomised controlled trial of trigeminal nerve stimulation for drug-resistant epilepsy. Neurology 2013;80:786-91.

33 Wu C, Sharan AD. Neurostimulation for the treatment of epilepsy: a review of current surgical interventions. Neuromodulation 2013;16:10-24.

34 Mormann F, Andrzejak RG, Elger CE, et al. Seizure prediction: the long and winding road. Brain 2007;130:314-33.

35 Morrell MJ. Responsive cortical stimulation for the treatment of medically intractable partial epilepsy. Neurology 2007;77:1295-304.

36 Nilsen K, Cock H. Focal treatment for refractory epilepsy: hope for the future?. Brain Res 2004;44:141-53.

37 Ludvig N, Medveczky J, French JA, et al. Evolution and prospects for intracrania pharmacotherapy for refractory epilepsies: the subdural hybrid neuroprosthesis. Epilepsy Res Treat 2010:2010:725696.

38 Engel J, McDermott M, Wiebe $S$, et al. Early surgical therapy for drug-resistant temporal lobe epilepsy: a randomised trial. JAMA 2012;307:922-30.

39 NICE clinical guideline 137. The epilepsies: the diagnosis and management of the epilepsies $\mathrm{n}$ adults and children in primary and secondary care. 2012.

40 Langfitt JT, Holloway RG, McDermott MP, et al. Health care costs decline after successful epilepsy surgery. Neurology 2007;68:1290-8. 